\title{
Gap structures and wave functions of classical waves in large-sized two-dimensional quasiperiodic structures
}

\author{
Y. Lai, ${ }^{1}$ Z. Q. Zhang, ${ }^{1}$ C. H. Chan, ${ }^{2}$ and L. Tsang ${ }^{2}$ \\ ${ }^{1}$ Department of Physics, Hong Kong University of Science and Technology, Clear Water Bay, Kowloon, Hong Kong \\ ${ }^{2}$ Department of Electronic Engineering, City University of Hong Kong, Kowloon, Hong Kong
}

(Received 8 May 2006; published 30 August 2006)

\begin{abstract}
By using the sparse-matrix canonical-grid method, we performed large-scale multiple-scattering calculations to study the gap structures and wave functions of classical waves in two-dimensional quasiperiodic structures. We observed many interesting phenomena arising from the quasiperiodic long-range order. In particular, a self-similar wave function with resonant structures was observed at a band edge. Our findings indicate that two-dimensional quasiperiodic systems exhibit a universal behavior that applies to both electrons (or phonons) in discrete lattices and classical waves in continuous media.
\end{abstract}

DOI: 10.1103/PhysRevB.74.054305 PACS number(s): 71.23.Ft, 43.40.+s, 42.70.Qs, 61.44.Br

\section{INTRODUCTION}

During the past two decades, a new class of materials called photonic crystals has attracted much research attention. ${ }^{1-5}$ Analogous to electrons in a crystal, electromagnetic waves propagating in photonic crystals are organized into photonic bands that are separated by gaps where propagating states are forbidden. Photonic crystals possess many interesting properties, such as complete spectral gaps, ${ }^{2}$ superprism, ${ }^{3}$ negative refraction, ${ }^{4}$ and self-collimation, ${ }^{5}$ etc. These properties could lead to novel applications, ${ }^{2}$ such as photonic crystal fibers and optical circuits, etc. Recently, research on photonic crystals has further extended to the propagation of other classical waves, such as elastic and/or acoustic waves ${ }^{6}$ and liquid surface waves, ${ }^{7}$ in periodic composites, which can exhibit similar wave properties as electromagnetic waves in photonic crystals.

The propagation of classical waves in quasiperiodic structures, which are analogs of electrons (or phonons) in quasicrystals, ${ }^{8,9}$ is another interesting and challenging topic that has attracted much attention recently. ${ }^{10-23}$ The study of quasiperiodic composites is much more difficult than that of periodic composites, due to the absence of the Bloch theorem. In previous studies, large photonic and/or phononic band gaps have been found in various quasiperiodic systems. ${ }^{10-17}$ Defects and waveguides, ${ }^{10,18}$ negative refraction, ${ }^{19}$ lasing at band edges, ${ }^{20}$ as well as nonlinear effects $^{21}$ have been studied in photonic quasicrystals. Most of these phenomena are also related to the formation of gaps in quasiperiodic systems. However, the system sizes used in previous theoretical studies were limited, usually to hundreds of cylinders in two dimensions, due to computational limitations. Thus, the previously obtained gap structures in two dimensions were mostly determined by the short-range order. How the quasiperiodic long-range order modifies the gap structures as well as the related phenomena observed in small samples is an important question. In low-contrast systems, the gap structures could be explained via the diffraction patterns (Born approximation), ${ }^{17,20}$ but this approach fails in high-contrast multiple-scattering systems. ${ }^{17}$ The possible interesting phenomena arising from the quasiperiodic long-range order remain almost unexplored. All these can only be answered by studying large samples.

In this paper, by using the sparse-matrix canonical-grid (SMCG) method, ${ }^{24}$ we have carried out first-principles multiple-scattering calculations to study the gap structures and wave functions of classical waves in large-sized twodimensional (2D) quasiperiodic structures. As the sample size was increased, we found new states emerging inside the original gaps and new gaps emerging inside the original bands, indicating a self-similar-like evolution in the gap structures. This self-similar-like evolution implies an unsmooth modification of the density of states due to the quasiperiodic long-range order in large samples, which, in turn, could strongly modify the phenomena observed in small samples. We also found the self-similar states as well as large-sized localized states. In particular, we identified a selfsimilar state with resonant structures at a band edge in a large sample containing 33919 cylinders. Our results originate from the quasiperiodic long-range order and indicate that 2D quasiperiodic systems exhibit a universal behavior that applies to both electrons (or phonons) in discrete lattices and classical waves in continuous media.

\section{PHYSICAL SYSTEM AND METHOD OF CALCULATION}

In this work, we study classical wave propagation in a 12-fold symmetric square-triangle tiling generated by the Stamfli inflation rule ${ }^{25}$ as shown in Fig. 1(a). Previous stud-

(a)

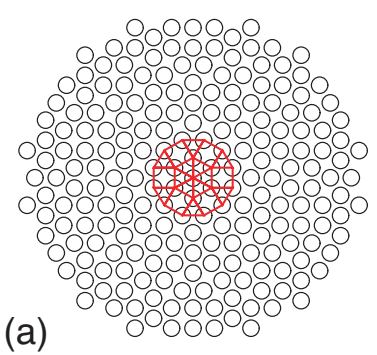

FIG. 1. (Color online) (a) The self-similar tiling sample of $R_{s}=8 a$. The seed dodecagon is plotted with red (dark gray) lines (b); the random tiling sample of $R_{s}=8 a$, which contains both the seed dodecagon [red (dark gray) lines] and that rotated by $30^{\circ}$ [blue (dark gray) dashed lines]. 
ies on such a structure in small-sized samples have shown large photonic and/or phononic band gaps due to both the short-range order and the high rotational symmetry. ${ }^{13-15}$ Here, we consider pressure waves in a composite of aluminum cylinders in an air host. The radii of the aluminum cylinders are $0.4 a$, where $a$ is the distance between two nearest cylinders. Due to the high density contrast between aluminum and air and a large filling fraction, the Born approximation is not applicable to our system. The transverse elastic waves in the aluminum cylinders can be ignored ${ }^{26}$ and we only need to consider the following scalar equation:

$$
-\frac{\omega^{2}}{\lambda} p=\nabla \cdot\left(\frac{1}{\rho} \nabla p\right)
$$

where $\omega$ is the frequency, $\lambda$ and $\rho$ are, respectively, the bulk modulus and density. The above equation can be mapped to the Maxwell equation for TM (or TE) waves if $\rho$ (or $\lambda$ ) is set as a constant. Here, Eq. (1) is solved by using the multiplescattering theory (MST). ${ }^{14}$ In order to calculate large systems, we have improved the program efficiency by incorporating the SMCG method ${ }^{24}$ into the MST method. The idea of the SMCG method is to introduce a canonical grid into the multiple-scattering system, so that the interactions between far-away cylinders can be calculated via the grid by using fast Fourier transform, thus resulting in an $N \log N$-type efficiency for CPU and $O(N)$ for memory, where $N$ is the number of scatterers in the system. In solving the coupled linear equations given by MST, we use the generalized minimal residue (GMRES) iterative method. ${ }^{27}$ In order to confirm convergence, we increased the cutoff angular quantum number in MST to 6 and reduced the normwise backward error in GMRES to $10^{-5}$.

\section{NUMERICAL RESULTS AND DISCUSSIONS}

We first investigate the evolution of gap structures as the sample size is increased. To do this, we place a monochromatic point source of frequency, $f$, near the center of a circular sample with radius $R_{s}$. Then, the gap structure can be obtained from the radiation power spectrum. The radiation power is calculated by integrating the total output energy flux and dividing it by the total flux without the sample. A band gap corresponds to a dip in the radiation power spectrum, which deepens as the sample size is increased. The radiation power spectrum has been proven accurate for the determination of gap positions in the previous literature. ${ }^{14} \mathrm{In}$ Fig. 2(a), we plot the calculated radiation power spectra in the frequency range of $0<\tilde{f}<1.2$ in dimensionless unit $\tilde{f}=f a / c_{a i r}\left(c_{a i r}\right.$ is the sound speed in air) for three samples of different radii, i.e., $R_{s}=3 a, 6 a$, and $8 a$. For comparison, we note that the radius of the seed dodecagon is $R_{\text {seed }} \approx 2 a$ in the square-triangle tiling and the inflation constant is $(2+\sqrt{3})$ $\approx 3.7$. When the sample size is increased from $R_{s}=3 a$ to $6 a$, the radiation power at some frequency ranges decreases significantly, indicating the positions of gaps, as indicated by the thick arrows in Fig. 2(a). However, when the sample size is increased to $R_{s}=8 a$, some new resonances emerge inside the original gaps, as indicated by the thin arrows in Fig. 2(a).

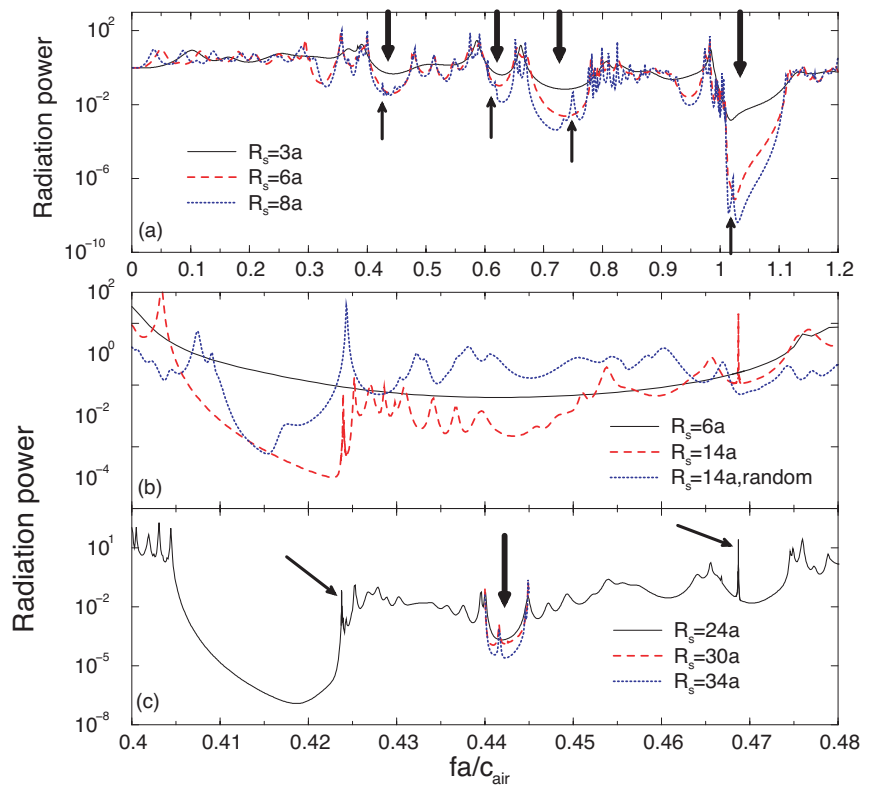

FIG. 2. (Color online) (a) The radiation power spectra for the quasiperiodic samples of radii $R_{s}=3 a$ (black curve), $6 a$ [red (dark gray) dashed curve], and $8 a$ [blue (dark gray) dotted curve]. (b) The radiation power spectra for quasiperiodic samples of radii $R_{s}=6 a$ (black curve) and $14 a$ [red (dark gray) dashed curve], and for a random sample of radius $R_{s}=14 a$ [blue (dark gray) dotted curve]. (c) The radiation power spectra for quasiperiodic samples of radii $R_{S}=24 a$ (black curve), $30 a$ [red (dark gray) dashed curve], and $34 a$ [blue (dark gray) dotted curve].

When the sample size is further increased to $R_{s}=14 a$, the radiation power spectrum is shown in Fig. 2(b) with a red (dark gray) dashed curve in an original gap of $0.4 \leq \tilde{f} \leqq 0.48$. It is clearly seen that the new resonances that emerge after $R_{s} \geqslant 8 a$ have now formed a band, which reduces the original gap to $0.405 \leq \tilde{f} \leqq 0.423$.

Besides new states emerging inside the original gaps, we have also observed new gaps emerging inside the original bands. To show this, in Fig. 2(c), we plot with a black curve the radiation power spectrum for a sample of $R_{s}=24 a$ in the range of $0.4 \leqslant \tilde{f} \leqslant 0.48$. By comparing this curve with that for $R_{s}=14 a$ in Fig. 2(b), we find a new dip around the frequency $\tilde{f}=0.442$, as indicated with a thick arrow in Fig. 2(c). In order to confirm this dip as a new gap, we calculate the radiation power spectra for even larger samples of $R_{s}=30 a$ and $34 a$ in the range of $0.44 \leqslant \tilde{f} \leqslant 0.445$, which are plotted as a red (dark gray) dashed curve and a blue (dark gray) dotted curve in Fig. 2(c), respectively. It is clearly seen that the dip deepens with sample size, indicating the existence of a new gap here. However, a new resonant state at $\tilde{f}=0.44165$ emerges inside this new gap when $R_{s} \geqslant 30 a$. Thus, we have observed a self-similar-like behavior of states emerging inside gaps and gaps emerging inside bands when the sample size is increased.

In addition to the radiation power spectrum, we have also calculated the average intensity $I(r)$ as a function of distance $r$ from the center of the sample, to study the properties of the wave functions. The average intensity $I(r)$ is obtained by 
integrating the intensity $|p|^{2}\left(\vec{r}^{\prime}\right)$ in an annular ring defined by $r-d r / 2<\left|\vec{r}^{\prime}\right|<r+d r / 2$ and dividing it by its area, $2 \pi r d r$, with $d r$ taken as $0.2 a$ in this work. It is known that, in a 2D periodic structure, wave functions are extended for frequencies inside a band and $I(r)$ is inversely proportional to $r$; while at a band edge, $I(r)$ exhibits a Bessel-function-like envelope, as a result of the Fabry-Perot effect. However, for each resonance shown in Fig. 2(c), the envelope of $I(r)$ is neither inversely proportional to $r$, nor in Bessel-functionlike form. Here, we show a particular band-edge state at $\widetilde{f}=0.4239$, which is indicated with a thin arrow in Fig. 2(c). This resonance first appears when $R_{s} \geqslant 8 a$, as shown in Fig. 2(a). Figure 3(a) shows the field intensity map $|p|^{2}(\vec{r})$ of this resonance for a large sample of $R_{s}=100 a$ consisting of 33919 cylinders. It is interesting to see that this intensity pattern is formed by many smaller ring patterns with radii of $R_{\text {ring }} \approx 7 a$, which almost coincide with the minimal sample size to yield this resonance in the spectrum. In Fig. 3(b), $I(r)$ is plotted on a log-log scale. The envelope of $I(r)$ explicitly exhibits a power-law behavior of $I(r) \propto 1 / r^{1.65}$. The powerlaw behavior is known to be the signature of a self-similar state in a quasiperiodic system. ${ }^{28,29}$ In Fig. 3(b), we also plot $I(r)$ for a smaller sample of $R_{s}=34 a$. However, with this sample size, it is difficult to differentiate the power-law decay from the exponential decay.

Another interesting resonance is at $\tilde{f}=0.4687$, which is also indicated with a thin arrow in Fig. 2(c). This resonance emerges when $R_{s} \geqslant 11 a$. In Fig. 3(c), we plot the intensity map $|p|^{2}(\vec{r})$ of this resonance for a sample of $R_{s}=34 a$, which contains 3925 cylinders. The corresponding $I(r)$ is plotted in Fig. 3(d) on a linear-log scale. It is interesting to see that the envelope of $I(r)$ is confined to a small core area with a radius of $R_{\text {core }} \approx 11 a$ with a flat tail outside. The size of the core area coincides with the minimal sample size, i.e., $11 a$, to yield this resonance in the spectrum. Another localized state is found at $\widetilde{f}=0.44165$ in the new gap indicated with the thick arrow in Fig. 2(c), when $R_{s} \geqslant 30 a$. This state has a core radius of about $29 a$, which is again consistent with the minimal sample size required to see this resonance. It should be pointed out that both localized states found here are supported by quasiperiodic long-range order. They emerge only when the sample size is sufficiently large. Thus, they are different from the localized states induced by the short-range order in small samples, ${ }^{30}$ where the wave functions are concentrated only in the first neighbors of the central cylinder.

The large-sized localized states found here can still be viewed as resonant excitations due to specific local environments. To confirm this point, we have randomized the cylinders' positions outside the core area and found that the resonances remain. According to Conway's theorem, ${ }^{28}$ when the sample size is sufficiently large, similar local environments can appear in the sample quasiperiodically. We cannot, therefore, exclude the possibility that the large-sized localized states found here will give rise to self-similar states in a much larger sample. From our observations, we propose the following coherent picture to describe the formation of a self-similar state in classical waves. As the sample size is increased, new local environments absent in smaller samples
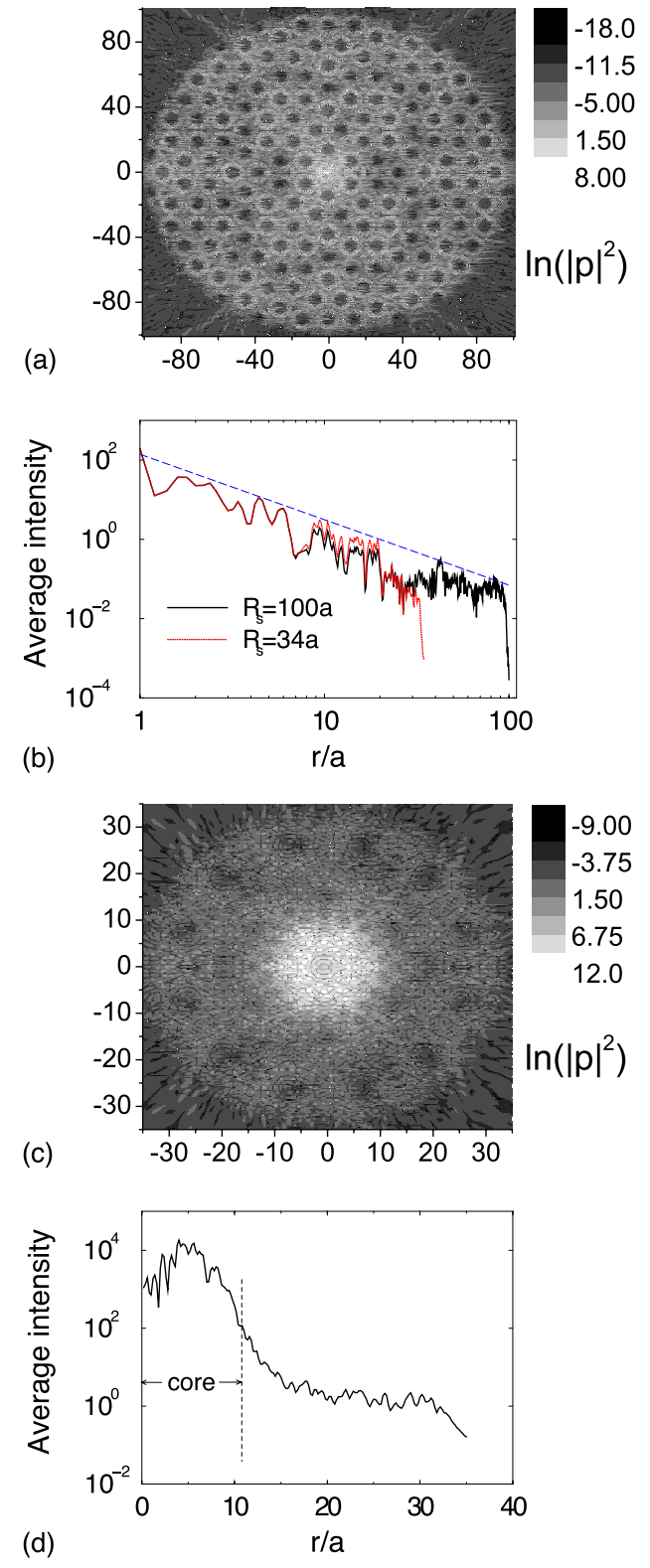

FIG. 3. (Color online) (a) The intensity distribution $|p|^{2}(\vec{r})$ (in $\log$ scale) of a band edge state at $f a / c_{\text {air }}=0.4239$ in a sample of $R_{s}=100 a$ containing 33919 cylinders. (b) The black curve shows the corresponding average intensity, $I(r)$, on the log-log scale for the resonance shown in (a). The blue (dark gray) dashed line shows the envelope of $I(r)$. The red (dark gray) dotted curve is the $I(r)$ in a smaller sample of $R_{s}=34 a$. (c) The intensity distribution $|p|^{2}(\vec{r})$ (in $\log$ scale) of a localized state at $f a / c_{\text {air }}=0.4687$ in a sample of $R_{s}=34 a$. (d) The corresponding average intensity $I(r)$ on the linear$\log$ scale for the resonance shown in (c). The core area is indicated in the graph.

are formed. These new local environments may support new localized resonances in the original gaps. As the sample size is further increased, these local environments repeat themselves quasiperiodically following Conway's theorem. The coupling of many localized resonances then forms a band inside the original gaps and the state at the band edge may result in a self-similar state. This picture explains the ringlike self-similar state found here. 
We have suggested that the interesting phenomena of the self-similar-like spectrum, the self-similar state, and largesized localized states are all due to the quasiperiodic longrange order. To directly verify this assertion, we have also calculated the radiation power spectrum of a random sample generated by allowing each offspring dodecagon to have $50 \%$ chance to rotate by $30^{\circ}$ from the seed (parent) dodecagon during the inflation process. ${ }^{25}$ In a random sample so generated, the long-range order is destroyed while the shortrange order inside the seed dodecagon is preserved, as is shown in Fig. 1(b). From the radiation power spectrum of a random sample, we find that the high-frequency gap structures are almost unchanged. However, the low-frequency gap structures are greatly influenced. In Fig. 2(b), we plot with a blue (dark gray) dotted curve the radiation power spectrum of a random sample of $R_{s}=14 a$. Clearly, the gap of $0.405 \lesssim \tilde{f} \lesssim 0.423$ found previously in a self-similar sample is destroyed after randomization, as well as the self-similar state at $\tilde{f}=0.4239$. Furthermore, both the localized states at $\tilde{f}=0.4687$ and $\tilde{f}=0.44165$ disappear in the random sample due to the destruction of long-range ordered local environments.

Finally, we note that the energy spectra and wave functions for electrons in quasicrystals have been extensively studied previously based on simple tight-binding (TB) models, which also included vibrational modes in the lattice. ${ }^{9}$ In a 1D Fibonacci lattice, it is found that the energy spectrum is singular continuous, consisting of a self-similar Cantor set with zero Lebesgue measure. ${ }^{31}$ Self-similar states exhibiting power-law behaviors may appear at band edges as well as band centers. In $2 \mathrm{D}$, the existence of self-similar wave functions with a power-law decay has also been conjectured ${ }^{28}$ and rigorously shown for the ground state in a Penrose lattice. ${ }^{29}$ All these distinctive phenomena are due to the quasiperiodic long-range order, as the interesting phenomena shown in this paper. However, it should be pointed out that classical waves are very different from TB electrons. In TB models, the transport of electrons is limited to the short- range hoppings. While for classical waves, the transport is conducted by the long-range radiation from each excited scatterer to all other scatterers. This naturally leads to the absence of "confined states" in classical waves, which have been observed for TB electrons in 2D. ${ }^{32}$ Moreover, in classical waves, the wave energy can occupy the host continuum as well as the scatterers. ${ }^{33}$ In our system of aluminum cylinders in an air host, the wave energy is concentrated in the air host. For the case of electromagnetic waves, it corresponds to a system of air cylinders in a dielectric host. These situations are opposite to the TB models, where electrons sit in the discrete lattices. Thus, it is not obvious that the phenomena found in TB models still apply to classical waves. The results we observed here strongly indicate the existence of a universal behavior for 2D quasiperiodic systems that applies to both electrons (or phonons) in discrete lattices and classical waves in continuous media.

\section{SUMMARY}

In conclusion, by using large-scale calculations, we have observed the interesting phenomena for classical waves in 2D quasiperiodic structures, including a self-similar-like evolution in the gap structures, a self-similar state at a band edge, and large-sized localized states. These findings arise from the quasiperiodic long-range order. Although we only studied a specific acoustic system in this work, we believe that similar phenomena exist for other classical waves, e.g., electromagentic waves in photonic quasicrystals, when the sample sizes are sufficiently large. Since the existence of band gaps as well as band-edge states is the basis of many applications in optics and/or acoustics, our results could also have important implications for applications.

\section{ACKNOWLEDGMENTS}

The authors thank C. T. Chan and P. Sheng for stimulating discussions. The work was supported by Hong Kong RGC Grant No. CA02/03.SC05.
${ }^{1}$ E. Yablonovitch, Phys. Rev. Lett. 58, 2059 (1987); S. John, ibid. 58, 2486 (1987).

${ }^{2}$ J. D. Joannopoulos, R. D. Meade, and J. N. Winn, Photonic Crystals (Princeton University Press, Princeton, 1995); Photonic Band Gap Materials, edited by C. M. Soukoulis (Kluwer, Dordrecht, 1996).

${ }^{3}$ H. Kosaka, T. Kawashima, A. Tomita, M. Notomi, T. Tamamura, T. Sato, and S. Kawakami, Phys. Rev. B 58, R10096 (1998).

${ }^{4}$ M. Notomi, Phys. Rev. B 62, 10696 (2000).

${ }^{5}$ H. Kosaka, T. Kawashima, A. Tomita, M. Notomi, T. Tamamura, T. Sato, and S. Kawakami, Appl. Phys. Lett. 74, 1212 (1999).

${ }^{6}$ M. M. Sigalas and E. N. Economou, J. Sound Vib. 158, 377 (1992).

${ }^{7}$ X. Hu, Y. Shen, X. Liu, R. Fu, J. Zi, X. Jiang, and S. Feng, Phys. Rev. E 68, 037301 (2003).

${ }^{8}$ D. Shechtman, I. Blech, D. Gratias, and J. W. Cahn, Phys. Rev. Lett. 53, 1951 (1984).
${ }^{9}$ The Physics of Quasicrystals, edited by P. J. Steinhardt and S. Ostlund (World Scientific, Singapore, 1987).

${ }^{10}$ Y. S. Chan, C. T. Chan, and Z. Y. Liu, Phys. Rev. Lett. 80, 956 (1998).

${ }^{11}$ C. Jin, B. Cheng, B. Man, Z. Li, D. Zhang, S. Ban, and B. Sun, Appl. Phys. Lett. 75, 1848 (1999).

${ }^{12}$ M. A. Kaliteevski, S. Brand, R. A. Abram, T. F. Krauss, R. DeLa Rue, and P. Millar, Nanotechnology 11, 274 (2000).

${ }^{13}$ M. E. Zoorob, M. D. B. Charlton, G. J. Parker, J. J. Baumberg, and M. C. Netti, Nature (London) 404, 740 (2000).

${ }^{14}$ X. Zhang, Z.-Q. Zhang, and C. T. Chan, Phys. Rev. B 63, 081105(R) (2001).

${ }^{15}$ Y. Lai, X. Zhang, and Z.-Q. Zhang, J. Appl. Phys. 91, 6191 (2002).

${ }^{16}$ M. Hase, H. Miyazaki, M. Egashira, N. Shinya, K. M. Kojima, and S. I. Uchida, Phys. Rev. B 66, 214205 (2002).

${ }^{17}$ A. Della Villa, S. Enoch, G. Tayeb, V. Pierro, V. Galdi, and F. 
Capolino, Phys. Rev. Lett. 94, 183903 (2005).

${ }^{18}$ M. Bayindir, E. Cubukcu, I. Bulu, and E. Ozbay, Phys. Rev. B 63, 161104(R) (2001).

${ }^{19}$ Z. Feng, X. Zhang, Y. Wang, Z.-Y. Li, B. Cheng, and D. Z. Zhang, Phys. Rev. Lett. 94, 247402 (2005).

${ }^{20}$ M. Notomi, H. Suzuki, T. Tamamura, and K. Edagawa, Phys. Rev. Lett. 92, 123906 (2004).

${ }^{21}$ R. Lifshitz, A. Arie, and A. Bahabad, Phys. Rev. Lett. 95, 133901 (2005)

${ }^{22}$ W. Man, M. Megens, P. J. Steinhardt, and P. M. Chaikin, Nature (London) 436, 993 (2005).

${ }^{23}$ L. Dal Negro, C. J. Oton, Z. Gaburro, L. Pavesi, P. Johnson, A. Lagendijk, R. Righini, M. Colocci, and D. S. Wiersma, Phys. Rev. Lett. 90, 055501 (2003).

${ }^{24}$ C. H. Chan and L. Tsang, Microwave Opt. Technol. Lett. 8, 114 (1995); L. Tsang, J. A. Kong, K. H. Ding, and C. O. Ao, Scattering of Electromagnetic Waves (Wiley, New York, 2001).
${ }^{25}$ P. Stampfli, Helv. Phys. Acta 59, 1260 (1986).

${ }^{26}$ M. Kafesaki and E. N. Economou, Phys. Rev. B 60, 11993 (1999).

${ }^{27}$ S. Yousef, Iterative Methods for Sparse Linear Systems (PWS Pub. Co., Boston, 1996).

${ }^{28}$ H. Tsunetsugu, T. Fujiwara, K. Ueda, and T. Tokihiro, Phys. Rev. B 43, 8879 (1991).

${ }^{29}$ B. Sutherland, Phys. Rev. B 34, 3904 (1986).

${ }^{30}$ Y. Wang, X. Hu, X. Xu, B. Cheng, and D. Zhang, Phys. Rev. B 68, 165106 (2003).

${ }^{31}$ M. Kohmoto, B. Sutherland, and C. Tang, Phys. Rev. B 35, 1020 (1987).

${ }^{32}$ T. Fujiwara, M. Arai, T. Tokihiro, and M. Kohmoto, Phys. Rev. B 37, 2797 (1988).

${ }^{33}$ M. Kafesaki and E. N. Economou, Phys. Rev. B 52, 13317 (1995). 